\title{
Protective effect of USP22 against paraquat-induced lung injury via activation of SIRT1/NRF2 pathway
}

\author{
Qiaosu Xiao¹, Rui Lu¹, Chunmei He ${ }^{1}$ Kai Zhou ${ }^{1, *}$
}

\begin{abstract}
${ }^{1}$ Department of Emergency Intensive Care Unit, the Affiliated Hospital of Southwest Medical University, 646000 Luzhou City, Sichuan Province, China
\end{abstract}

\section{*Correspondence}

zhoukai_999@163.com

(Kai Zhou)

\begin{abstract}
Introduction: The nonselective herbicide Paraquat $(\mathrm{PQ})$ is broadly used in agricultural production. However, $\mathrm{PQ}$ has severe toxicity in humans and results in over $90 \%$ of death due to lack of effective therapy strategies. Ubiquitin Specific Peptidase 22 (USP22) is a deubiquitinase and it exerts a vital role in regulating ROS production. This study aimed to study the effect of USP22 on PQ-induced lung injury and investigate the precise mechanism.

Methods: The lung injury model was induced by treating with PQ. Hematoxylin and eosin (HE) staining and lung wet/dry ratio were conducted to assess lung tissue injury. Myeloperoxidase (MPO) activity was detected to evaluate neutrophil infiltration in the lung tissues. Superoxidase dismutase (SOD) activity and Malondialdehyde (MDA) content were measured to determine oxidative damage. Cell viability and cell apoptosis were detected using MTT assay and Flow cytometry.

Results: PQ caused lung tissue damage and increased lung wet/dry ratio. PQ increased the MPO activity and MDA content, and decreased SOD activity. USP22 was downregulated in PQ-treated mice. Besides, overexpression of USP22 alleviated PQ-induced cell apoptosis and oxidative damage in vitro. Furthermore, overexpression of USP22 increased the expression of Sirtuin 1 (SIRT1)/nuclear factor E2-related factor 2 (NRF2). Down-regulation of SIRT1 reversed the beneficial influence of overexpressed USP22 on PQ-induced cell apoptosis and oxidative damage. Moreover, overexpression of USP22 attenuated PQ induced lung injury in vivo.

Conclusions: Overexpression of USP22 alleviated PQ-induced lung injury through activating SIRT1/NRF2 pathway. USP22 may be a valuable target for the treatment of PQ-induced lung injury.
\end{abstract}

\section{Keywords}

Paraquat; Lung injury; USP22; SIRT1; NRF2; Oxidative damage

\section{Introduction}

Paraquat (PQ), a nonselective herbicide, is broadly utilized in agricultural production worldwide due to high efficiency and low price [1]. However, PQ has severe toxicity in animals and humans. Previous studies report that PQ enters the body via inhalation, contact, and ingestion [2], which results in the injury of body organs such as lungs, liver, and kidneys [1,3]. Among the organs, the lung is the main damage target [4]. Paraquat can lead to PQ lung, and the early symptoms are acute lung injury (ALI) or acute respiratory distress syndrome (ARDS), and the later symptoms appear as fibrosis in the alveoli and pulmonary interstitial [5]. Currently, the morbidity of PQ poisoning remains high, and it caused more than $90 \%$ of death because of ineffective therapy strategies [6]. Therefore, it is essential to investigate the PQ poisoning mechanism, especially in lung injury, and explore therapeutic strategies with high clinical efficacy.
Previous studies revealed that the leading cause of PQ toxicity was ROS accumulation [6, 7]. In lung tissues, PQ was reduced to free radicals by NADPH assisted single-electron reduction, and then hydrogen peroxide is produced by superoxide dismutase (SOD), and the more toxic hydroxyl radical also was formed. Excessive accumulation of ROS could damage lipid, protein and deoxyribonucleic acid, and other macromolecular substances, thereby damaging cell membrane and cell structure $[3,8]$. Besides, ROS produced by PQ also affected cell viability and apoptosis [1]. Hence, therapy related to antioxidant and anti-apoptosis may be a promising strategy for PQ poisoning treatment.

Ubiquitin specific proteinase 22 (USP22) belongs to the deubiquitinase family, and it functions as an oncogene in many cancers [9-12]. For instance, Liu et al. [9] found that USP22 was associated with liver metastasis and poor prognosis of colorectal cancer. Besides, USP22 was demonstrated to attenuate myocardial and intestinal $\mathrm{I} / \mathrm{R}$ injury [13, 14]. In myocardial 
I/R injury, elevated USP22 repressed ROS generation and lipid peroxidation [13]. Furthermore, USP22 could suppress apoptosis, ROS generation, and inflammation caused by high glucose in podocytes [15]. Therefore, we inferred that USP22 might exert an antitoxic effect on PQ poisoning. Hence, we investigated the role of USP22 in PQ-induced lung injury and explored the mechanism of the action.

\section{Materials and methods}

\subsection{Animals}

BALB/C mice were acquired from Beijing Vital River Laboratory Animal Technology (Beijing, China). All mice were grouped into sham group, paraquat (PQ) group, lenti-vector group, lenti-USP22 group, lenti-vector + PQ group, and lentiUSP22 + PQ group. Six mice were allocated to each group. The mice in the PQ group were intraperitoneally injected with $50 \mathrm{mg} / \mathrm{kg}$ paraquat. The PQ dosage was selected according to the previous studies $[16,17]$. The mice in the sham group were intraperitoneally injected with the same amount of normal saline. In the lenti-vector group, lenti-USP22 group, lenti-vector + PQ group, or lenti-USP22 + PQ group, mice were intratracheally injected with lentivirus vector or lentivirus carrying USP22 gene 7 days before they were treated with normal saline or PQ. The animal experiments were carried out following the national and international regulations and policies. The Committee of Animal Experimentation of the Affiliated Hospital of Southwest Medical University approved the study.

\subsection{Cell culture}

Mouse lung epithelial cell line MLE-12 was acquired from the American Type Culture Collection (ATCC). MLE-12 cells were maintained in DMEM medium plus 10\% fetal bovine serum (FBS) (Gibco, Carlsbad, CA, USA) at $37{ }^{\circ} \mathrm{C}$ with $5 \%$ $\mathrm{CO}_{2}$. In indicated experiments, MLE-12 cells were treated with $200 \mu \mathrm{M}$ paraquat [18, 19] or $20 \mu \mathrm{M}$ sirtinol [20, 21] for $24 \mathrm{~h}$.

\subsection{Lung wet/dry ratio}

After the mice were sacrificed, lung tissues were collected. The left lung was excised and the wet weight was recorded. The tissues were placed into oven at $60{ }^{\circ} \mathrm{C}$ for $72 \mathrm{~h}$ to remove water. Afterward, the dry tissues weight was determined, and the ratio of wet weight to dry (W/D) weight was analyzed.

\subsection{HE staining}

Lung tissues were harvested, fixed using paraformaldehyde, embedded by paraffin, and sectioned into $5 \mu \mathrm{m}$ samples. The samples were stained with hematoxylin and eosin, following by examination under a light microscope (Olympus, Tokyo, Japan).

\subsection{Determination of myeloperoxidase and superoxidase dismutase activity and malondialdehyde (MDA) content}

After the mice were sacrificed, lung tissues were collected to prepare lung homogenates. After the homogenates were centrifuged, the supernatant was used to determine the activity of myeloperoxidase (MPO) and superoxidase dismutase (SOD) and the content of malondialdehyde (MDA) using the commercial kits (Abcam, Cambridge, MA, UK) following the manufacturer's procedures.

\subsection{Western blot}

The lysates of lung tissues and MLE-12 cells were prepared using RIPA buffer (Thermo Fisher Scientific, Waltham, MA, USA) and quantified through a BCA kit (Sigma, St. Louis, MO, USA). Subsequently, the lysates were subjected to SDSPAGE and transferred to PVDF membranes. The membranes were probed with anti-USP22 (1 : 1000), Sirtuin 1 (SIRT1) (1 : 2000), nuclear factor E2-related factor 2 (NRF2) (1 : $1000)$, and $\beta$-actin $(1: 1000)$ antibodies (Abcam, Cambridge, $\mathrm{MA}, \mathrm{UK})$ at $4{ }^{\circ} \mathrm{C}$ for $12 \mathrm{~h}$ after blocking by $4 \%$ non-fat milk. Then, the membranes were incubated with secondary antibody IgG H\&L (HRP) (Abcam, Cambridge, MA, UK). The bands were imaged utilizing the ECL Plus Western Blotting Substrate (Thermo Fisher Scientific, Waltham, MA, USA). $\beta$-actin was used as a reference protein.

\subsection{3-(4,5-Dimethylthiazol-2-yl)-2,5- diphenyltetrazolium bromide assay}

To determine cell viability, 3-(4,5-Dimethylthiazol-2-yl)-2,5diphenyltetrazolium bromide (MTT) assay was conducted. MLE-12 cells were plated into 96-wells plates. After $24 \mathrm{~h}$ of culture, cells were treated with $20 \mu \mathrm{L}$ MTT $(5 \mathrm{mg} / \mathrm{mL})$ (Sigma, St. Louis, MO, USA) at $37^{\circ} \mathrm{C}$ for $4 \mathrm{~h}$. Subsequently, $100 \mu \mathrm{L}$ Dimethyl Sulfoxide (DMSO) was mixed to the cells to remove crystallization. The microplate reader recorded the absorbance at $490 \mathrm{~nm}$ of each well.

\subsection{Flow cytometry}

Cell apoptosis determination was conducted using Annexin VFITC Apoptosis Detection Kit (Sigma, St. Louis, MO, USA). MLE-12 cells were collected through digestion by trypsin and washed using PBS. Collected cells were resuspended using binding buffer and incubated with Annexin-V and PI for 15 mins without light. Afterward, Flow Cytometry (BD, San Jose, CA, USA) was employed to quantify the apoptotic cells.

\subsection{Cell transfection}

USP22 overexpression plasmid, sh-SIRT1, and corresponding controls were acquired from Genscript (Genscript, Nanjing, China). To perform cell transfection, MLE-12 cells were plated into 6-wells plates and maintained at $37{ }^{\circ} \mathrm{C}$ for 24 h. Subsequently, MLE-12 cells were transfected with USP22 plasmid, sh-SIRT1, or corresponding controls by Lipofectamine 2000 (Invitrogen, Carlsbad, CA, USA) following the 
A

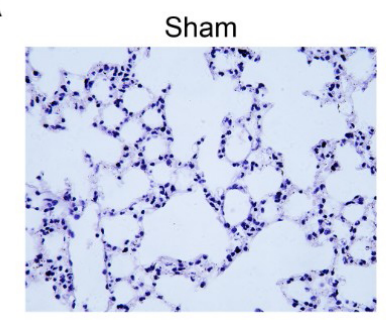

D

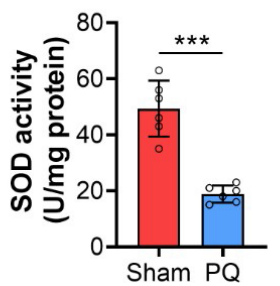

B

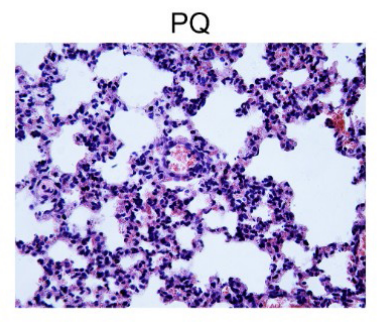

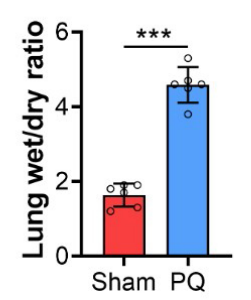

C

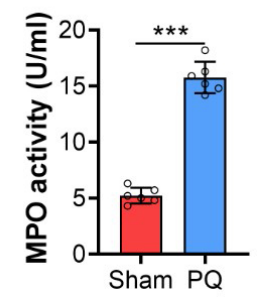

F I G U R E 1. Paraquat (PQ) induced lung injury and decreased USP22 expression. (A) HE staining was performed to assess lung injury in mice treated with PQ. (B) Lung wet/dry ratio was determined in mice treated with PQ. (C) The activity of MPO in lung tissues was measured in mice treated with PQ. (D) The activity of SOD and the content of MDA were measured in lung tissues of mice treated with PQ. (E) Western blot was conducted to determine the protein level of USP22 in lung tissues of mice treated with PQ. ${ }^{* * *}: p<0.001$.

protocol of the manufacturer. These cells were harvested to conduct subsequent experiments $48 \mathrm{~h}$ later.

\subsection{Statistical analysis}

Data were displayed as mean \pm standard deviation (SD). Data analysis was completed using SPSS Statistics 22.0 (SPSS, Chicago, IL, USA). One-way ANOVA with LSD's post hoc test or Student's $t$-test was employed to assess statistical significance with $p<0.05$ considered as statistically significant.

\section{Results}

\subsection{PQ induced lung injury and decreased USP22 expression}

To induce lung injury, the mice were intraperitoneally injected with $50 \mathrm{mg} / \mathrm{kg}$ PQ. After 7 days, HE staining was performed to assess lung injury. Results revealed that PQ caused lung tissue damage, reflected by small and irregular alveolar, thickened alveolar wall and inflammatory cells infiltrating compared to the sham group (Fig. 1A). The lung wet/dry ratio was increased in mice treated by PQ compared to sham mice $(p<0.001$, Fig. 1B). In addition, the activity of MPO was measured to evaluate the infiltration of neutrophils and macrophages in lung tissues. MPO activity was enhanced in PQ-treated lung tissues $(p<0.001$, Fig. 1C). Furthermore, $\mathrm{PQ}$ treatment decreased the activity of SOD $(p<0.001)$ and increased the MDA content in lung tissues $(p<0.001$, Fig. 1D). Moreover, USP22 was significantly inhibited in lung tissues of mice treated by $\mathrm{PQ}$ compared to sham mice ( $p<0.001$, Fig. 1E). Therefore, PQ induced lung injury and decreased USP22 expression.

\subsection{Elevated USP22 alleviated PQ-induced cell apoptosis and oxidative damage}

To understand the influence of USP22 on PQ-induced lung injury, the USP22 overexpression plasmid was introduced into MLE-12 cells 7 days before PQ treatment. The transfection efficiency of the USP22 overexpression plasmid in MLE-12 cells was confirmed using western blot. Results suggested that USP22 was significantly elevated in MLE12 cells after transfection $(p<0.001$, Fig. 2A). Besides, the decreased expression of USP22 induced by PQ in MLE12 cells was reversed by the USP22 overexpression plasmid $(p<0.01$, Fig. 2A). Overexpressed USP22 did not affect the viability of MLE-12 cells (Fig. 2B). However, the cell viability was decreased after treatment of PQ $(p<0.001)$, which was abrogated by elevated USP22 ( $p<0.01$, Fig. 2 B). Furthermore, PQ treatment induced apoptosis of MLE-12 cells $(p<0.001)$, which was abrogated by enhanced USP22 expression $(p<0.01$, Fig. 2C,D). However, USP22 did not affect cell apoptosis of MLE-12 cells in the absence of PQ (Fig. 2C,D). Moreover, the decreased SOD activity in cells treated by PQ was abolished by enhanced USP22 expression $(p<0.01$, Fig. 2E). Overexpression of USP22 decreased the elevated MDA content in PQ-treated cells ( $p<0.05$, Fig. 2E). Thus, elevated USP22 alleviated PQ-induced cell apoptosis and oxidative damage.

\subsection{Elevated USP22 increased the expression of SIRT1 and NRF2}

SIRT1/NRF2 signaling was considered an effective target for the antioxidant therapy of $\mathrm{PQ}$ poisoning [22]. Besides, a study revealed that USP22 inhibited the ubiquitination of SIRT1 and stabilized the SIRT1 expression [23]. Therefore, SIRT1/NRF2 signaling might participate in the regulation of USP22 on 


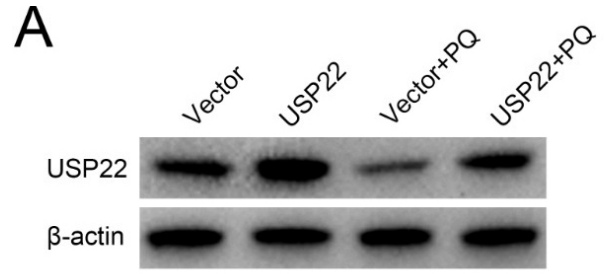

C

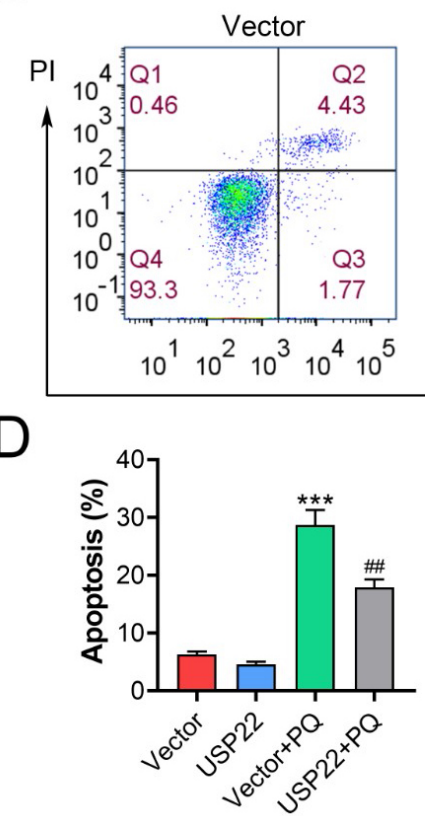

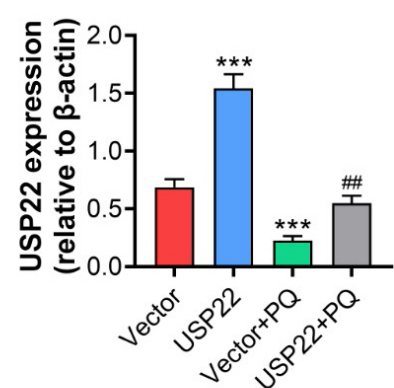

USP22

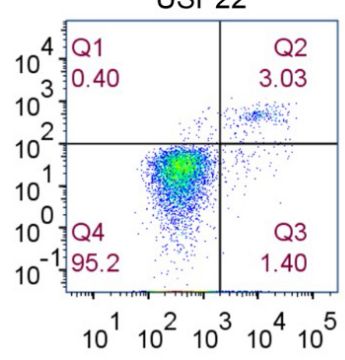

B

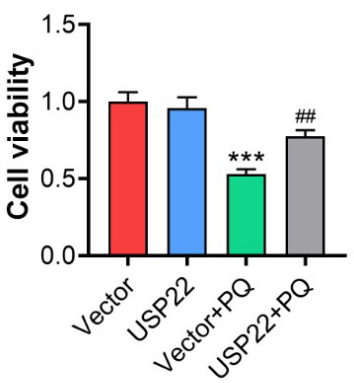

$\mathrm{E}$

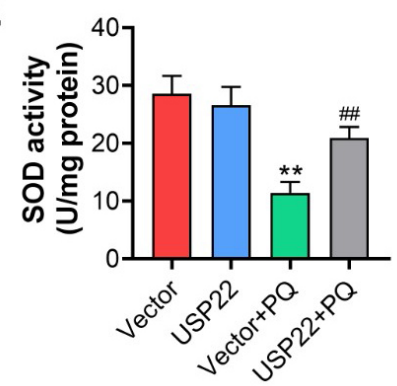

Vector $+P Q$

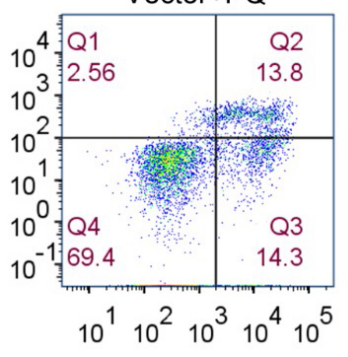

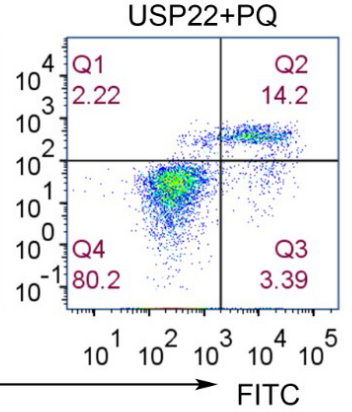

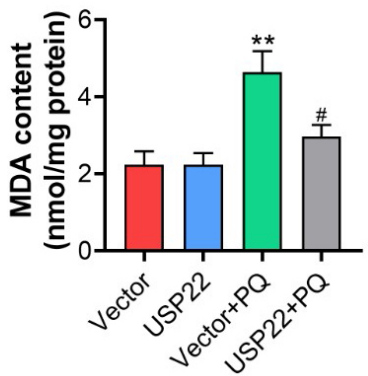

F I G U R E 2. Overexpression of USP22 attenuated PQ-induced cell apoptosis and oxidative damage. (A) The protein level of USP22 in MLE-12 cells transfected with USP22 vector and treated with PQ was determined using Western blot. (B) Viability of MLE-12 cells after USP22 vector transfection and PQ treatment was evaluated using MTT assay. (C) and (D) Apoptosis of MLE-12 cells after transfected with USP22 vector and treated with PQ was determined by Flow cytometry. (E) The activity of SOD and the content of MDA were measured in MLE-12 cells transfected with USP22 vector and treated with PQ. ${ }^{* *}: p<0.01$; $* * *: p<0.001 . \#: p<0.05$; \#\#: $p<0.01$.

PQ-induced injury. Therefore, to elucidate the underlying regulatory mechanism of USP22 on PQ-induced cell apoptosis and oxidative damage, the protein levels of SIRT1 and NRF2 were determined after overexpression of USP22 in MLE-12 cells. Results showed that overexpression of USP22 greatly enhanced the levels of SIRT1 $(p<0.001)$ and NRF2 $(p<$ $0.01)$ in MLE-12 cells (Fig. 3). In MLE-12 cells treated by $\mathrm{PQ}$, the expression levels of SIRT1 $(p<0.001)$ and NRF2 $(p<0.01)$ were remarkably decreased and overexpression of USP2 2 abrogated these decrease $(p<0.01$, Fig. 3$)$. These results revealed that overexpression of USP 22 upregulated the expression of SIRT1 and NRF2.

\subsection{Down-regulation of SIRT1 reversed the protective effect of elevated USP22 on PQ-induced cell apoptosis and oxidative damage}

To better understand the function of the SIRT1/NRF2 pathway on the regulation process of USP22 on PQ-induced cell apoptosis and oxidative damage, the sh-SIRT1 was transfected into the MLE-12 cells treated by PQ, and the inhibitor of SIRT1 sirtinol was also used to treat the cells. Western blot results showed that overexpression of USP22 abrogated the inhibitory effects of PQ on SIRT1 and NRF2 in MLE-12 cells, which was abolished by knockdown of SIRT1 (all $p<0.001$, Fig. 4A). Besides, the viability of MLE-12 cells was suppressed by PQ ( $p$ $<0.001$ ), but overexpression of USP22 increased the viability of MLE-12 cells ( $p<0.01$, Fig. 4B). However, both sh-SIRT1 and sirtinol significantly abolished the effect of USP22 on cell viability (two $p<0.01$, Fig. 4B). In addition, down-regulation of SIRT1 also abolished the protective effect of overexpressing USP22 on PQ-induced cell apoptosis of MLE-12 cells (all $p$ $<0.05$, Fig. 4C,D). Moreover, SOD activity was decreased, and MDA content was increased in PQ-treated MLE-12 cells (two $p<0.01$ ), which were reversed by overexpressing USP22 (two $p<0.01$, Fig. 4E). However, down-regulation of SIRT1 abolished the effects of USP22 on SOD activity and MDA content (all $p<0.05$, Fig. 4E). Hence, down-regulation of SIRT1 reversed the protective effects of USP22 overexpression on PQ-induced cell apoptosis and oxidative damage. Together these results suggest SIRT1 participated in the regulation of USP22 on PQ-induced injury. 

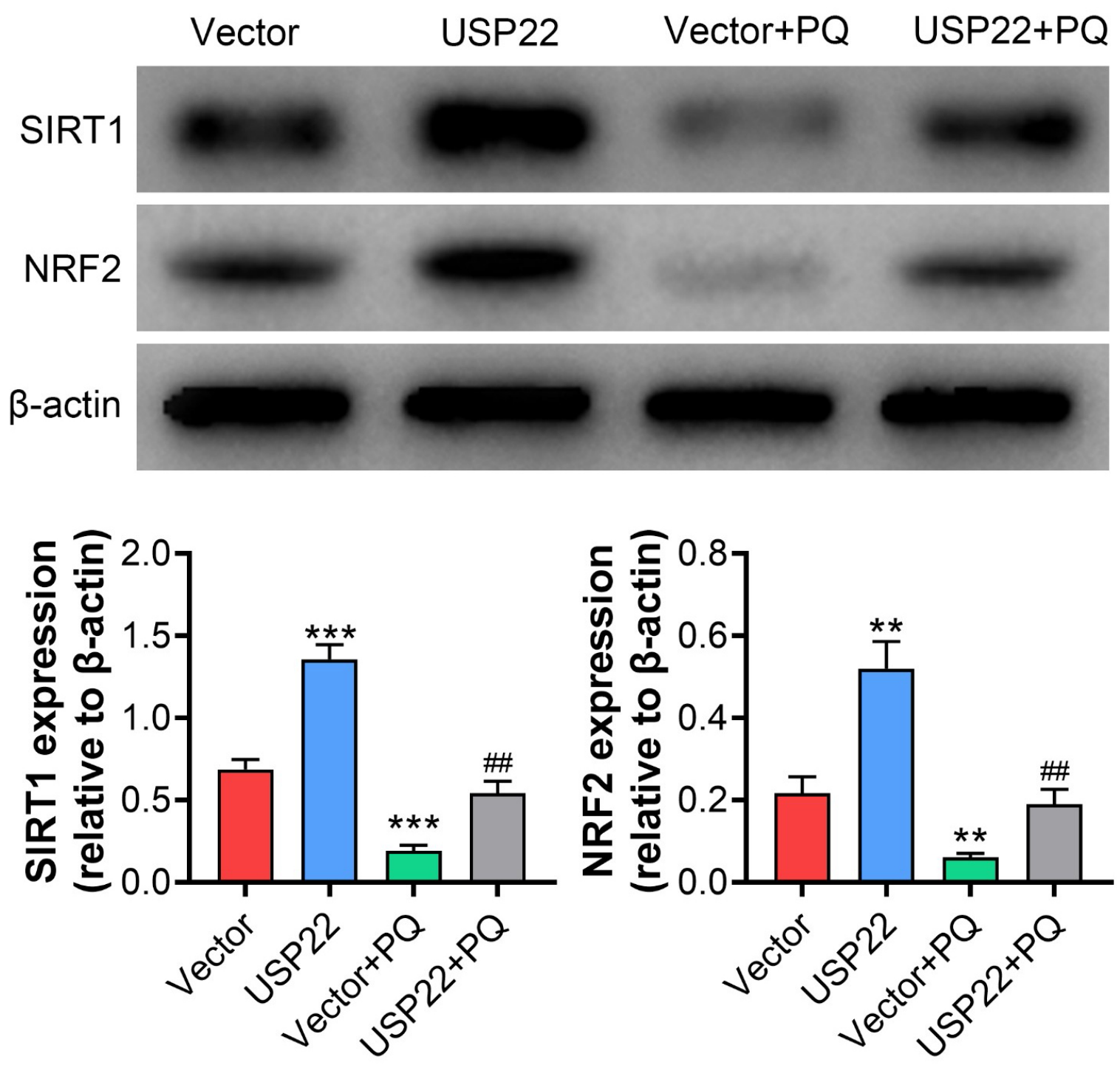

F I G URE 3. Overexpression of USP22 upregulated the expression of SIRT1 and NRF2. The protein levels of SIRT1 and NRF2 were determined using Western blot in MLE-12 cells after USP22 vector transfection and PQ treatment. **: $p<0.01$; ***: $p<0.001$. \#\#: $p<0.01$.

\subsection{Elevated USP22 alleviated PQ-induced lung injury}

To further study the effect of USP22 on PQ-induced lung injury, the mice were intratracheally injected with lentivirus carrying the USP22 gene before PQ treatment. Then, the elevated expression level of USP22 was verified using western blot in mice ( $p<0.001$, Fig. 5A). HE staining results revealed that overexpression of USP22 alleviated the lung injury induced by PQ (Fig. 5B). Besides, the lung wet/dry ratio was increased in mice treated by PQ $(p<0.001)$ but was decreased by elevated USP22 ( $p<0.001$, Fig. 5 C). The increased MPO activity in lung tissues of mice treated by PQ was inhibited by overexpressed USP22 (all $p<0.001$, Fig. 5D). Furthermore, the decreased SOD activity and increased MDA content in lung tissues of mice treated by PQ were reversed by overexpressing USP22 (all $p<0.001$, Fig. 5E). Moreover, the decreased levels of SIRT1 and NRF2 in lung tissues of mice treated by PQ were abrogated by overexpressing USP22 (all $p<0.001$, Fig. 5F). Taken together, overexpression of USP22 attenuated
PQ-induced lung injury.

\section{Discussion}

Paraquat (PQ) is broadly used in agricultural production to control weed growth, while the drug has high toxicity to humans [1]. It can lead to injury of body organs, especially the lung [4]. The mortality rate of PQ poisoning is over $90 \%$ [6]. Thus, it is imperative to search for an effective therapeutic strategy for PQ poisoning. In this study, we studied the role of USP22 in the PQ-induced lung injury and revealed that overexpression of USP22 attenuated PQ-induced lung injury via activating the SIRT/NRF2 pathway.

To establish the lung injury model, PQ was used to treat mice. After PQ treatment, the mice's alveolar became small and irregular, and the lung wet/dry ratio was increased. Besides, MPO activity was increased in lung tissues of mice treated by PQ. Previous studies reported that MPO activity was considered the biomarker to evaluate neutrophil infiltration in the lung tissue $[24,25]$. PQ was also proved to induce ROS 
A
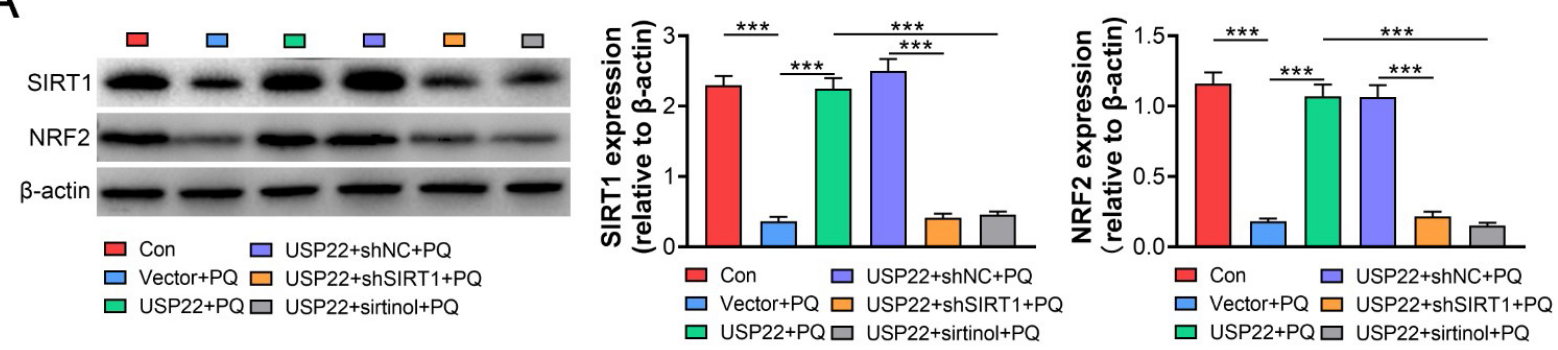

B

$\mathrm{C}$

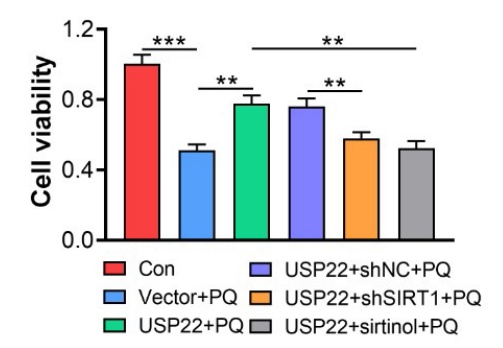

D

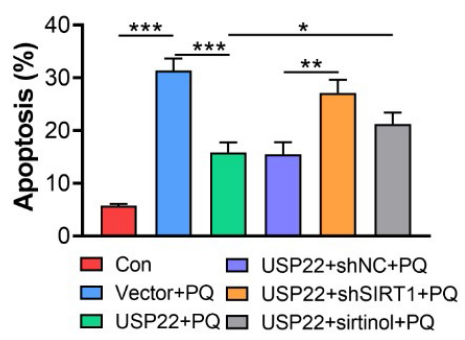

$E$
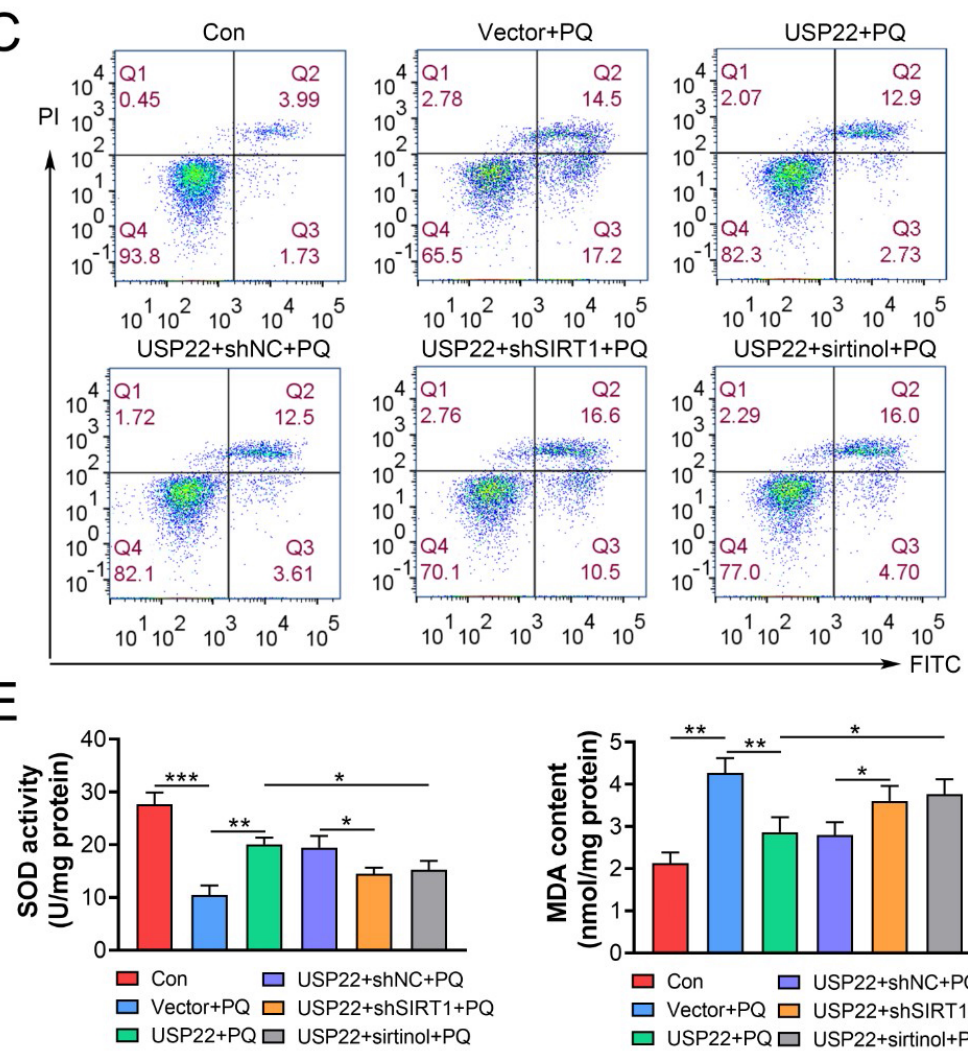

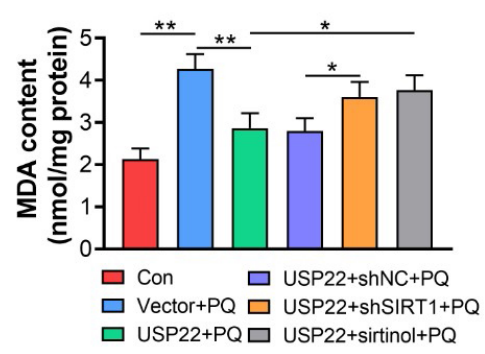

F I G URE 4. Down-regulation of SIRT1 reversed the protective effect of USP22 overexpression on PQ-induced cell apoptosis and oxidative damage. (A) The protein levels of SIRT1 and NRF2 in MLE-12 cells treated with PQ and transfected with USP22 vector and sh-SIRT1 were determined using western blot. (B) Viability of MLE-12 cells treated with PQ and transfected with USP22 vector and sh-SIRT1 was determined using MTT assay. (C) and (D) Apoptosis of MLE-12 cells treated with PQ and transfected with USP22 vector and sh-SIRT1 was assessed using Flow cytometry. (E) The activity of SOD and the content of MDA were measured in MLE-12 cells treated with PQ transfected with USP22 vector and sh-SIRT1. *: $p<0.05 ; * *$ : $p<0.01 ; * *: p<0.001$.

production and cause oxidative damage to tissues and cells in animals and humans $[26,27]$. In this study, we found that PQ decreased the activity of SOD and increased MDA content. These results indicated that PQ successfully induced lung injury.

As a deubiquitination protein, USP22 regulates ROS production in myocardial $\mathrm{I} / \mathrm{R}$ injury and high glucose-induced cell injury $[13,15]$, which implies that USP22 might regulate PQ-induced lung injury. To determine the role of USP22 in PQ-induced lung injury, USP22 expression in lung tissues of mice treated by PQ was determined. Results revealed that USP22 was decreased in the lung tissues of mice treated by PQ. Besides, overexpression of USP22 decreased PQ-induced oxidative damage in MLE-12 cells, consistent with the previous studies $[13,15]$. In addition, PQ suppress cell viability and induced cell apoptosis in many tissues and cells, such as human neural progenitor cells and lung cancer cells [28, 29]. Therefore, the roles of USP22 in cell viability and apoptosis were examined in MLE-12 cells after PQ treatment. We found that overexpression of USP22 reversed the PQ-induced decrease in cell viability and decreased the PQ-induced cell apoptosis of MLE-12 cells. Hi et al. [14] also reported that elevated USP22 promotes cell proliferation in intestinal epithelial cells after hypoxia/reoxygenation injury. Zhou et al. [30] showed that down-regulation of USP22 promoted apoptosis of human retinoblastoma cells. Therefore, these results were in agreement with the previous reports [14, 30]. Furthermore, our findings revealed that overexpression of USP22 could inhibit lung tissue injury and oxidative damage in PQ-treated mice in vivo. The function of USP22 on PQ-induced lung injury was 


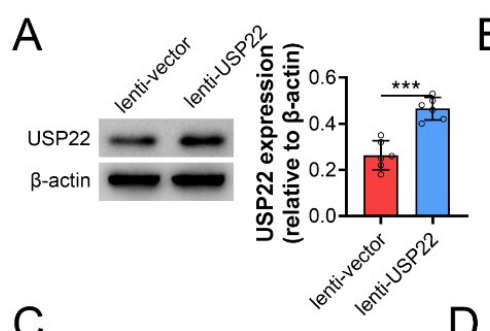

C

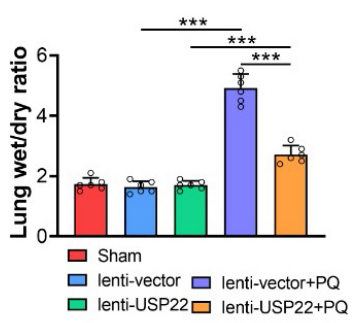

$\mathrm{F}$

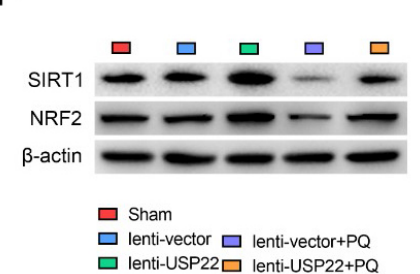

B

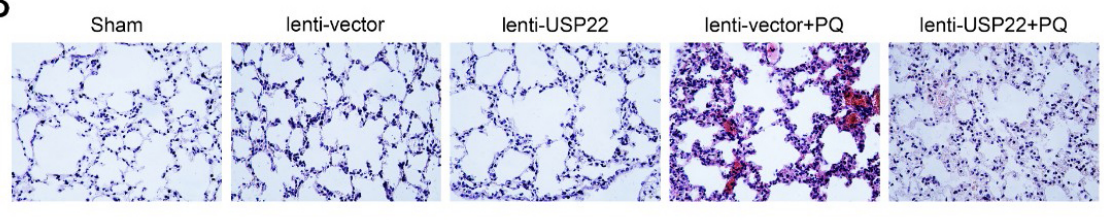

$E$
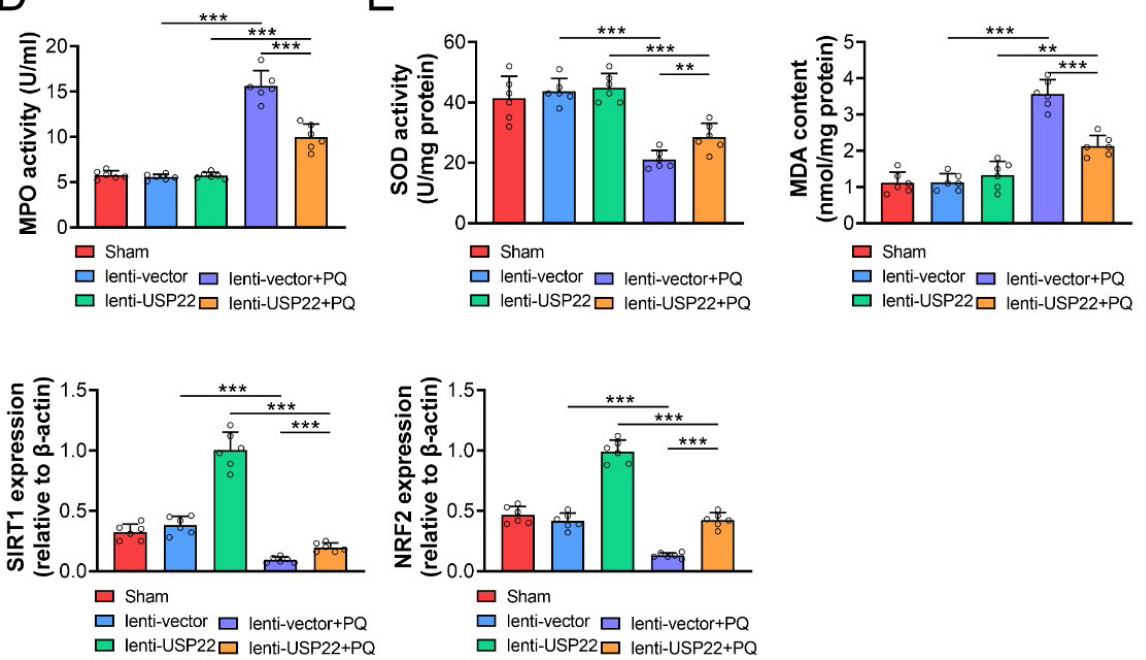

F I G U R E 5. Overexpression of USP22 attenuated PQ-induced lung injury. (A) Western blot was used to determine the expression of USP22 in mice injected with lentivirus carrying USP22 and treated by PQ. (B) HE staining was conducted to assess lung injury in mice with overexpression of USP22 and PQ treatment. (C) Lung wet/dry ratio was analyzed in mice with overexpression of USP22 and PQ treatment. (D) The activity of MPO in lung tissues was determined in mice with overexpression of USP22 and PQ treatment. (E) The activity of SOD and the content of MDA were determined in mice with overexpression of USP22 and PQ treatment. (F) Western blot was conducted to determine the protein levels of SIRT1 and NRF2 in lung tissues of mice with overexpression of USP22 and PQ treatment. **: $p<0.01 ; * *: p<0.001$.

demonstrated in the study for the first time.

Sirtuin 1 (SIRT1)/nuclear factor E2-related factor 2 (NRF2) signaling pathway plays vital functions in PQ poisoning treatment [22]. In addition, USP22 could inhibit the ubiquitination of SIRT1 and stabilize the expression of SIRT1 [23]. Furthermore, USP22 inhibits apoptosis and promotes cell proliferation through antagonizing p53 function through the regulation of SIRT1 [23]. Thus, we inferred that the SIRT1/NRF2 signaling might participate in the regulation of USP22 on PQinduced lung injury. Results revealed that overexpression of USP22 upregulated the levels of SIRT1 and NRF2, and downregulation of SIRT1 reversed the protective effect of USP22 overexpression on PQ-induced cell apoptosis and oxidative damage, which was consistent with the study conducted by Lin et al. [23]. NRF2 signaling is a vital pathway in cellular response to oxidative stress-induced injury [31,32]. In normal condition, NRF2 is in an inactive state through binding to Keap1 (a negative regulator of NRF2). When stimulated, NRF2 dissociates from Keap1, translocates to the nucleus, and binds to Maf, thereby regulating the expression of anti-oxidant genes in stimulation state [33, 34]. SIRT1 exerts crucial roles in regulating apoptosis and oxidative stress [35, 36]. Besides, Han et al. [37] proved that SIRT1 could activate the NRF2 pathway and then SIRT1/NRF2 signaling mediates the protective effect of dietary melatonin on chromiuminduced lung injury. Thus, we inferred that USP22 might protect PQ-induced through elevating the expression of SIRT1, and increased SIRT1 activates NRF2 pathway and induces the expression of anti-oxidant genes, thereby alleviating cell apoptosis and oxidative damage. Taken together, the beneficial effect of USP22 on PQ-induced lung injury is exerted via activating the SIRT1/NRF2 pathway.

\section{Conclusions}

Paraquat induced lung tissue injury and oxidative damage and decreased the expression of USP22. Overexpression of USP22 attenuated PQ-induced cell apoptosis and oxidative damage in vitro and in vivo, and increased the expression of SIRT1 and NRF2. Besides, the down-regulation of SIRT1 reversed the protective effect of elevated USP22 on PQ-induced cell apoptosis and oxidative damage. In summary, overexpression of USP22 alleviated PQ-induced lung injury through activating SIRT1/NRF2 pathway. Taken together, USP22 may be a valuable target for the treatment of PQ-induced lung injury.

\section{AUTHOR CONTRIBUTIONS}

QSX and RL designed the study, supervised the data collection. $\mathrm{CMH}$ analyzed the data, interpreted the data. $\mathrm{KZ}$ prepare the manuscript for publication and reviewed the draft of the manuscript. All authors have read and approved the 
manuscript.

\section{ETHICS APPROVAL AND CONSENT TO PARTICIPATE}

Ethical approval was obtained from the Ethics Committee of the Affiliated Hospital of Southwest Medical University (Approval No.2020892).

\section{ACKNOWLEDGMENT}

Thanks to all the peer reviewers for their opinions and suggestions.

\section{FUNDING}

This research received no external funding.

\section{CONFLICT OF INTEREST}

The authors declare no conflict of interest.

\section{AVAILABILITY OF DATA AND MATERIALS}

All data generated or analyzed during this study are included in this published article.

\section{REFERENCES}

[1] Zhang Z, Nian Q, Chen G, Cui S, Han Y, Zhang J. Klotho alleviates lung injury caused by paraquat via suppressing ROS/P38 MAPK-regulated inflammatory responses and apoptosis. Oxidative Medicine and Cellular Longevity. 2020; 2020: 1854206.

[2] Yin Y, Guo X, Zhang SL, Sun CY. Analysis of paraquat intoxication epidemic (2002-2011) within China. Biomedical and Environmental Sciences. 2013; 26: 509-512.

[3] Dinis-Oliveira RJ, Duarte JA, Sánchez-Navarro A, Remião F, Bastos ML, Carvalho F. Paraquat poisonings: mechanisms of lung toxicity, clinical features, and treatment. Critical Reviews in Toxicology. 2008; 38: 1371.

[4] Zhang L, Li Q, Liu Z, Liu W, Zhao M. Protective effects of a rho kinase inhibitor on paraquat-induced acute lung injuries in rats. Inflammation. 2018; 41: 2171-2183.

[5] Chen C, Chou H, Hsu H, Wang L. Transforming growth factor- $\beta 1$ upregulation is independent of angiotensin in paraquat-induced lung fibrosis. Toxicology. 2005; 216: 181-187.

[6] Ren Y, Yang Z, Sun Z, Zhang W, Chen X, Nie S. Curcumin relieves paraquat-induced lung injury through inhibiting the thioredoxin interacting protein/NLR pyrin domain containing 3-mediated inflammatory pathway. Molecular Medicine Reports. 2019; 20: 5032-5040.

[7] Liu B, Cao B, Zhang D, Xiao N, Chen H, Li G, et al. Salvianolic acid $\mathrm{B}$ protects against paraquat-induced pulmonary injury by mediating Nrf2/Nox 4 redox balance and TGF- $\beta 1 /$ Smad 3 signaling. Toxicology and Applied Pharmacology. 2016; 309: 111-120.

[8] Tomita M, Okuyama T, Katsuyama H, Miura Y, Nishimura Y, Hidaka K, et al. Mouse model of paraquat-poisoned lungs and its gene expression profile. Toxicology. 2007; 231: 200-209.

[9] Liu Y, Yang Y, Xu H, Dong X. Aberrant expression of USP22 is associated with liver metastasis and poor prognosis of colorectal cancer. Journal of Surgical Oncology. 2011; 103: 283-289.

[10] Schrecengost RS, Dean JL, Goodwin JF, Schiewer MJ, Urban MW, Stanek TJ, et al. USP22 regulates oncogenic signaling pathways to drive lethal cancer progression. Cancer Research. 2014; 74: 272-286.
[11] Zhang Y, Yao L, Zhang X, Ji H, Wang L, Sun S, et al. Elevated expression of USP22 in correlation with poor prognosis in patients with invasive breast cancer. Journal of Cancer Research and Clinical Oncology. 2011; 137: 1245-1253.

[12] Xu J, Tan Q, Li T. USP22 promotes the expression of GLUT1 and HK2 to facilitate growth and glycolysis in cervical cancer cells. European Journal of Gynaecological Oncology. 2020; 41: 790-796.

[13] Ma S, Sun L, Wu W, Wu J, Sun Z, Ren J. USP22 protects against myocardial ischemia-Reperfusion injury via the SIRT1-p53/SLC7A11dependent inhibition of ferroptosis-induced cardiomyocyte death. Frontiers in Physiology. 2020; 11: 551318.

[14] Ji A, Li T, Zu G, Feng D, Li Y, Wang G, et al. Ubiquitin-specific protease 22 enhances intestinal cell proliferation and tissue regeneration after intestinal ischemia reperfusion injury. World Journal of Gastroenterology. 2019; 25: 824-836.

[15] Shi J, Wang Q, Li H, Huang Q. Silencing of USP22 suppresses high glucose-induced apoptosis, ROS production and inflammation in podocytes. Molecular BioSystems. 2016; 12: 1445-1456.

[16] Li A, Liu Y, Zhai L, Wang L, Lin Z, Wang S. Activating peroxisome proliferator-activated receptors (PPARs): a new sight for chrysophanol to treat paraquat-induced lung injury. Inflammation. 2016; 39: 928-937.

[17] Tyagi N, Dash D, Singh R. Curcumin inhibits paraquat induced lung inflammation and fibrosis by extracellular matrix modifications in mouse model. Inflammopharmacology. 2016; 24: 335-345.

[18] Jang YJ, Won JH, Back MJ, Fu Z, Jang JM, Ha HC, et al. Paraquat Induces Apoptosis through a Mitochondria-Dependent Pathway in RAW264.7 Cells. Biomolecules \& Therapeutics. 2015; 23: 407-413.

[19] Sun D, Song C, Xu Y, Wang R, Liu W, Liu Z, et al. Involvement of PINK1/Parkin-mediated mitophagy in paraquat-induced apoptosis in human lung epithelial-like a549 cells. Toxicology in Vitro. 2018; 53: 148-159.

[20] Park SY, Kim HY, Park HJ, Shin HK, Hong KW, Kim CD. Concurrent treatment with taxifolin and cilostazol on the lowering of $\beta$-amyloid accumulation and neurotoxicity via the suppression of P-JAK2/PSTAT3/NF-кB/BACE1 signaling pathways. PLoS ONE. 2016; 11 : e0168286.

[21] Lee HR, Shin HK, Park SY, Kim HY, Lee WS, Rhim BY, et al. Attenuation of $\beta$-amyloid-induced tauopathy via activation of CK $2 \alpha / \mathrm{SIRT} 1$ : targeting for cilostazol. Journal of Neuroscience Research. 2014; 92: 206-217.

[22] Ding Y, Zhao G, Li X, Hong G, Li M, Qiu Q, et al. SIRT1 exerts protective effects against paraquat-induced injury in mouse type II alveolar epithelial cells by deacetylating NRF2 in vitro. International Journal of Molecular Medicine. 2016; 37: 1049-1058.

[23] Lin Z, Yang H, Kong Q, Li J, Lee S, Gao B, et al. USP22 antagonizes p53 transcriptional activation by deubiquitinating Sirt1 to suppress cell apoptosis and is required for mouse embryonic development. Molecular Cell. 2012; 46: 484-494.

[24] Kumari A, Tyagi N, Dash D, Singh R. Intranasal curcumin ameliorates lipopolysaccharide-induced acute lung injury in mice. Inflammation. 2015; 38: 1103-1112.

[25] An X, Sun X, Hou Y, Yang X, Chen H, Zhang P, et al. Protective effect of oxytocin on LPS-induced acute lung injury in mice. Scientific Reports. 2019; 9: 2836.

[26] Chen Q, Zhang X, Zhao J, Lu X, Zheng P, Xue X. Oxidative damage of the male reproductive system induced by paraquat. Journal of Biochemical and Molecular Toxicology. 2017; 31: e21870.

[27] Shalaby MA, Emam SR, Soliman AM. Protective effect of vitamin e against herbicide paraquat-induced enzymatic leakage and oxidative damage in the liver of rats. Advances in Animal and Veterinary Sciences. 2020; 8: 639-646.

[28] Chang X, Lu W, Dou T, Wang X, Lou D, Sun X, et al. Paraquat inhibits cell viability via enhanced oxidative stress and apoptosis in human neural progenitor cells. Chemico-Biological Interactions. 2013; 206: 248-255.

[29] Zerin T, Kim Y, Hong S, Song H. Protective effect of methylprednisolone on paraquat-induced a549 cell cytotoxicity via induction of efflux transporter, P-glycoprotein expression. Toxicology Letters. 2012; 208 : 101-107.

[30] Zhou D, Liu P, Sun D, Chen Z, Hu J, Peng S, et al. USP22 down-regulation facilitates human retinoblastoma cell aging and apoptosis via inhibiting 
TERT/P53 pathway. European Review for Medical and Pharmacological Sciences. 2017; 21: 2785-2792.

[31] Liu B, Zhang H, Tan X, Yang D, Lv Z, Jiang H, et al. GSPE reduces leadinduced oxidative stress by activating the Nrf2 pathway and suppressing miR153 and GSK-3 $\beta$ in rat kidney. Oncotarget. 2017; 8: 42226-42237.

[32] Tan X, Liu B, Lu J, Li S, Baiyun R, Lv Y, et al. Dietary luteolin protects against $\mathrm{HgCl}_{-} 2$-induced renal injury via activation of Nrf2-mediated signaling in rat. Journal of Inorganic Biochemistry. 2018; 179: 24-31.

[33] Lv Y, Jiang H, Li S, Han B, Liu Y, Yang D, et al. Sulforaphane prevents chromium-induced lung injury in rats via activation of the Akt/GSK3 $\beta$ /Fyn pathway. Environmental Pollution. 2020; 259: 113812.

[34] Yang D, Lv Z, Zhang H, Liu B, Jiang H, Tan X, et al. Activation of the Nrf2 signaling pathway involving klf9 plays a critical role in allicin resisting against arsenic trioxide-induced hepatotoxicity in rats. Biological Trace Element Research. 2017; 176: 192-200.

[35] Bai X, Fan L, He T, Jia W, Yang L, Zhang J, et al. SIRT1 protects rat lung tissue against severe burn-induced remote ALI by attenuating the apoptosis of PMVECs via p38 MAPK signaling. Scientific Reports. 2015; 5: 10277.

[36] Kim J, Jo J, Kim K, An H, Gwon M, Gu H, et al. Pharmacological activation of SIRT1 ameliorates cisplatin-induced acute kidney injury by suppressing apoptosis, oxidative stress, and inflammation in mice. Antioxidants. 2019; 8: 322.

[37] Han B, Li S, Lv Y, Yang D, Li J, Yang Q, et al. Dietary melatonin attenuates chromium-induced lung injuryviaactivating the Sirt1/Pgc$1 \alpha /$ Nrf2 pathway. Food \& Function. 2019; 10: 5555-5565.

How to cite this article: Qiaosu Xiao, Rui Lu, Chunmei He, Kai Zhou. Protective effect of USP22 against paraquat-induced lung injury via activation of SIRT1/NRF2 pathway. Signa Vitae. 2021;17(3):187-195. doi:10.22514/sv.2021.077. 